Discussion Two general classes of biological agents could be recognized. The first comprised infectious diseases, including but not limited to zoonotic infections. The second class comprised organisms resulting in the production of bioaerosols, thus increasing the occupational risk of immune-related and respiratory conditions. Some occupations (e.g. mine work and welding) might increase susceptibility of workers to infection, without increasing the exposure to this pathogen.

\section{S-85 SEX AND GENDER INEQUALITIES: SEGREGATION OF OHS EXPOSURES AND PREVENTIVE AVENUES FOR A POPULATION OF LOW-EDUCATED TEENAGERS ENTERING THE LABOUR FORCE}

${ }^{1}$ Marie Laberge, Aurélie Tondoux, Myriam Bérubé. 'University of Montreal, Canada

10.1136/OEM-2021-EPI.404

As men and women hold different jobs, have different social roles and different power influence in societal strata, they are exposed to different physical and psychosocial hazards at work. Adolescents with lower levels of education who start working are particularly vulnerable to sex/gender segregation of tasks and exposure to different work hazards.

In Quebec, adolescents who have experienced significant academic delays are referred to the Work-Oriented Training Program (WOTP). In this program, they learn semi-skilled trades by doing practicums, through Co-operative Education. These placements involve many occupational health and safety risks. For example, students may be exposed to various toxic substances in cleaning jobs, to wood or metal dust in processing plants or garages, to allergens in pet care businesses, hair salons, or child care centers. As jobs are segregated by gender, prevention approaches must take this into account. Occupational health and safety (OHS) risk factors may differ by sex and/or gender (e.g. when a small girl uses tools designed for tall men; or when manual handling training only considers 'boxes' as potential loads rather than a variety of situations, such as angry children or objects). Accordingly, occupational safety and health programs and prevention strategies should consider sex and gender-related factors. Our team is developing educational tools and resources to help these students improve and maintain their health as they enter the workforce, through an equity perspective. This presentation will discuss the differentiated hazards faced by low-educated male and female adolescents, as well as promising prevention avenues, including specific consideration for 'invisible risks' often encountered by young women. The WOTP examples that will be given can be applicable to other contexts of vocational training and work integration in low-skilled jobs for young workers.

\section{S-89 RESPIRATORY HEALTH IMPACTS OF CLEANING AND DISINFECTING EXPOSURES: DOES EVIDENCE SUPPORT A LINK WITH COPD?}

'Orianne Dumas, Nicole Le Moual. 'INSERM, CESP, France

\subsection{6/OEM-2021-EPI.405}

Introduction Concerns regarding the respiratory health effects of disinfectants and cleaning products (DCP) have been growing in the last two decades. Studies have shown associations between exposure to DCP and asthma. The irritant properties of many chemicals contained in DCP have prompted research on respiratory effects beyond asthma.

Objective to review the current evidence for an association between exposure to DCP and Chronic Obstructive Pulmonary Disease (COPD).

Methods We will present an overview of the recent epidemiological literature on the association between exposure to DCP at the workplace or at home and COPD. We will discuss challenges and opportunities for future research on this question.

Results Increased risk of chronic bronchitis or COPD have been reported among workers regularly exposed to DCP, such as cleaners or healthcare workers in both European and US populations. In a large cohort study of US female nurses, occupational exposure to DCP was significantly associated with an increased risk of developing COPD, independent of asthma. High-level exposure, evaluated by a job-task exposure matrix, to several specific disinfectants (e.g., glutaraldehyde, bleach, hydrogen peroxide) was associated with COPD incidence. An impact of exposure to DCP on lung function outcomes has also been reported in a European populationbased study. Exposure to cleaning activities either at work or at home was associated with accelerated FEV1 and FVC decline.

Conclusion Despite accumulating evidence for adverse effects of DCP on COPD, specific tasks and substances at risk still need to be elucidated. Exposure assessment methods need to be improved in epidemiological studies. Novel statistical approaches, such as the ones developed in the context of exposome research, may help disentangling the mutual effect of the numerous chemicals contained in DCP, as well as their mixture. The mechanisms underlying the association between DCP and respiratory health outcomes need to be clarified.

\section{S-92 GENDER/SEX DIFFERENCES IN WORKERS' COMPENSATION FOR HEARING LOSS, CONCUSSIONS AND ACTIVITY-RELATED SOFT TISSUE DISORDER (ASTDS) IN BRITISH COLUMBIA, CANADA}

${ }^{1}$ Mieke Koehoorn, Lillian Tamburic, Robert Macpherson, Sonja Senthanar, Chris McLeod. 'University of British Columbia, Canada

\subsection{6/OEM-2021-EPI.406}

Introduction Novel access to workers' compensation claims data, in particular disallowed claims and claim eligibility status decisions, was used to inform an evidence need on sex/gender differences in experiences of the workers' compensation process in British Columbia, Canada.

Objectives To conduct a sex/gender stratified analyses of the risk of work-related acute injuries and cumulative disorders, and of the adjudication of the work-relatedness of these injuries and disorders.

Methods Compensation claims for ASTDs, concussions and noise-induced hearing loss (2003-17) were analysed for differences between women and men by age-adjusted rates of accepted claims within occupations (ASTDs and concussions only), for ratios of disallowed to accepted claims (ASTDS and hearing loss only), and for time to final claim eligibility decision (ASTDs, concussions and hearing loss).

Results We observed higher rates and rates of increase for women compared to men in the same occupations for ASTDs 